\title{
cmaJOPEN
}

\section{Pregnant people's responses to the COVID-19 pandemic: a mixed-methods, descriptive study}

\author{
Hamideh Bayrampour MSc PhD, Sukhpreet K. Tamana PhD, Amelie Boutin PhD
}

Abstract

Background: Given the extent of the COVID-19 pandemic and uncertainty around the timing of its containment, understanding the experiences and responses of the perinatal population is essential for planning responsive maternity care both during and after the pandemic. The aim of this study was to explore the experiences of pregnant people and their responses to the COVID-19 pandemic, and to identify how health care providers can support this population.

Methods: This was a mixed-methods, descriptive study with cross-sectional and qualitative descriptive components. We conducted the study between Mar. 20 and May 31, 2020, in British Columbia, Canada. Any pregnant person at any gestational age living in BC was eligible to participate. We collected quantitative data using online survey tools, including the Edinburgh Postnatal Depression Scale (EPDS) and the 7-item Generalized Anxiety Disorder questionnaire (GAD-7). We collected qualitative data using open-ended questions to explore people's thoughts, feelings and experiences during the pandemic. Participants were recruited using study posters distributed via prenatal care clinics and classes, LifeLabs and social media across the province. We used thematic and descriptive analyses to analyze the data, and we integrated the qualitative and quantitative findings at the interpretation level.

Results: The study sample included 96 participants with mean ( \pm standard deviation) maternal and gestational ages of $32 \pm$ 3.92 years and $22.73 \pm 8.93$ weeks, respectively. Most $(93 \% ; n=89)$ identified as female. Of the participants, $54 \%(n=50)$ and $35 \%$ $(n=34)$ reported anxiety and depressive symptoms, as measured by the GAD-7 and EPDS, respectively. Overarching themes that emerged from the qualitative data included uncertainty about birth plans and setting, added burden to existing health and social disparities, perceived or projected lack of support or limited support, concerns about early development, and struggle over managing multiple demands. Perceived maternity care needs included mental health support, maintaining prenatal care, frequent and proactive check-ins to build rapport, and recommendations specific to the pandemic.

Interpretation: We found that the impact of the COVID-19 pandemic on the pregnant population has been substantial. The findings of this mixed-methods study can be used to help plan informed and evidence-based health care interventions to mitigate adverse effects and support mothers and families.

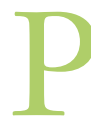

andemics cause extensive social, psychological, economic and political disruptions, and they increase morbidity and mortality. ${ }^{1,2}$ Analyses of the susceptibility of pregnant people to COVID-19 suggest that they have increased vulnerability as a result of physiologic alterations in the respiratory, circulatory and immune systems, and reproductive hormone changes associated with pregnancy. ${ }^{3}$ Findings of a systematic review of 19 studies on pregnancy and perinatal outcomes of pregnant people with coronavirusspectrum infections (including SARS-CoV-2) showed higher rates of preterm birth, miscarriage, preeclampsia, cesarean delivery and perinatal death compared to pregnant people who were not infected. ${ }^{4}$ In a United Kingdom cohort of pregnant people admitted to hospital with COVID-19 between Mar. 1 and Apr. 14, 2020, 10\% needed respiratory support in a critical care setting and the case fatality rate was $1.2 \% .^{5}$ Another study in France showed increased maternal morbidity and preterm birth and a $24.1 \%$ need for oxygen support among 54 pregnant people with confirmed or sus- pected COVID-19 between Mar. 1 and Apr. 3, 2020. ${ }^{6}$ Among noninfected women, a 2021 systematic review showed a significant increase in stillbirth and maternal mortality during the pandemic compared to before the pandemic. ${ }^{7}$

Historical data from the 1918 influenza pandemic, severe acute respiratory syndrome (SARS) and other infectious disease outbreaks show that even if pregnant people are not infected, they suffer disproportionately during such outbreaks. ${ }^{8-10}$ Pregnant people are often vigilant about the health and safety of their fetuses. A pandemic could intensify this

\section{Competing interests: None declared.}

This article has been peer reviewed.

Correspondence to: Hamideh Bayrampour, hamideh.bayrampour@ubc.ca

CMAJ Open 2022 February 22. DOI:10.9778/cmajo.20210136 
apprehension, because poor clinical outcomes among infected pregnant people ${ }^{11}$ and higher rates of pregnancy loss and preterm births have been reported in pandemics. ${ }^{4,10,12,13}$ Some medications for infectious diseases can also be harmful to the fetus., ${ }^{3,8}$ Moreover, media coverage of the mortality and morbidity associated with pregnancy can intensify fear among pregnant people. ${ }^{8}$

The perinatal population are major users of health care services in Canada. ${ }^{14}$ Pandemics may alter the capacity of health care systems to support routine health care services, including pregnancy, intrapartum and postpartum care. ${ }^{15} \mathrm{In}$ British Columbia, Canada, following the declaration of a state of emergency on Mar. 18, 2020, most inpatient nonemergency health services and surgeries were postponed, and outpatient services were offered via virtual care when possible.

Given the extent of the COVID-19 pandemic and uncertainty about the timing of its containment, understanding the experiences and responses of the perinatal population is essential for planning responsive maternity care services both during and after the pandemic. The aim of this study was to explore the experiences of pregnant people and their responses to the COVID-19 pandemic, and to identify how health care providers can support this population.

\section{Methods}

\section{Design and setting}

This was a mixed-methods, descriptive study with cross-sectional and qualitative descriptive components. We used a pragmatism framework to conduct the study. In this framework, qualitative and quantitative approaches are considered compatible and the primary emphasis is on the research question. ${ }^{16}$ This study was reported in accordance with the Consolidated criteria for reporting qualitative research (COREQ) checklist. ${ }^{17}$

The data for this study were collected as part of the Pregnancy Specific Anxiety Scale (PSAS) study, which was ongoing in $\mathrm{BC}$ when the COVID-19 pandemic emerged in Canada. The PSAS study was originally designed to develop a screening tool to assess pregnancy-specific anxiety, and it was implemented in 3 phases. The first case of community transmission of COVID19 in BC was confirmed on Mar. 5, 2020, and the BC government declared a state of emergency on Mar. 18, 2020. The current study involved adapting phase 3 of the PSAS study and used data collected between Mar. 20 and May 31, 2020. The goal of phase 3 was instrument evaluation and psychometric testing of the newly developed PSAS to establish its reliability and validity.

\section{Participants}

We recruited participants during their pregnancy using study posters distributed in prenatal care clinics and classes (the clinics were identified through Google search), LifeLabs across the province and social media (including Facebook, Twitter and Instagram). Any pregnant person at any gestational age living in $\mathrm{BC}$ was eligible to participate.

\section{Data sources}

After signing a consent form, participants completed an online survey to provide quantitative and qualitative data.

\section{Quantitative}

We collected detailed descriptive information on individual, obstetric and psychosocial characteristics using an online survey in REDCap (https://redcap.ubc.ca). We assessed depressive symptoms using the Edinburgh Postnatal Depression Scale (EPDS), ${ }^{18}$ a widely used, valid and reliable screening tool for perinatal depression; a cut-off score of 10 is recommended for community samples..$^{19} \mathrm{We}$ assessed anxiety symptoms using the 7-item Generalized Anxiety Disorder (GAD-7) questionnaire, ${ }^{20}$ a valid and reliable instrument developed to screen for generalized anxiety disorder and its severity.

\section{Qualitative}

As part of the online survey, participants responded to 6 openended questions in writing to describe their thoughts, feelings and experiences during the pandemic (Appendix 1, available at www.cmajopen.ca/content/10/1/E146/suppl/DC1). The research team developed these questions with the aim of capturing whether and how the outbreak had affected participants' everyday life; their physical and mental well-being, as well as the well-being of their pregnancy and baby; how they coped; and how their health care provider(s) could support them better during the pandemic. We imposed no word limit on the answer fields.

\section{Data analysis}

\section{Quantitative analysis}

We described individual, obstetric and mental health characteristics using proportions, means and measures of dispersion calculated using IBM SPSS Statistics version $25 .{ }^{21}$

\section{Qualitative analysis}

We used a qualitative descriptive approach ${ }^{22}$ to analyze qualitative data. This approach is appropriate for addressing health care practice and policy inquiries where researchers stay close to the data to describe and interpret findings in everyday terms. Two female researchers (H.B., S.T.) carried out a thematic analysis ${ }^{23}$ : H.B. is an assistant professor with research experience in qualitative and mixed-methods studies, and S.T. is a research associate with a doctoral qualification in clinical psychology. S.T. also conducted clinical diagnostic interviews with participants over the phone as part of the overall PSAS study after they had completed the online survey.

The analysts open-coded each participant's input after reading it. Codes were clustered into categories (subthemes) and then grouped into themes. The themes and categories were formulated to achieve a joint interpretation of the data.

The qualitative data were managed using Microsoft Excel. Analytic rigour (i.e., visibility of research practice and accountability of data analysis $)^{24,25}$ was ensured by independent analysis of the data by the researchers; by including participants' reflections and verbatim quotations; and by reporting participants' characteristics to enable readers to identify the study's applicability to diverse populations. 
Integration of qualitative and quantitative components Integration of the qualitative and quantitative components occurred at the interpretation level by embedding and merging the study findings and conclusions.

\section{Ethics approval}

This study was approved by the University of British Columbia Conjoint Health Research Ethics Board (H21-00489).

\section{Results}

The study sample included 96 pregnant participants with a mean ( \pm standard deviation) age of $32 \pm 3.92$ years. Gestational age varied between 7 and 40 weeks (mean \pm standard deviation $22.73 \pm 8.93$ weeks; Table 1$)$. All participants $(n=96)$ completed the anxiety and depression questionnaires (EPDS and GAD-7). All participants answered open-ended questions 1 to 4 . Eighteen participants did not answer open-ended questions 5 or 6 .

The findings of the qualitative analysis are grouped under 3 overarching themes: responses to the COVID-19 pandemic; participants' experiences related to the pandemic; and perceived maternity care needs (Table 2).

\section{Responses to the COVID-19 pandemic}

Two categories emerged from participants' responses to the pandemic: psychological responses, and responses to pandemicimposed prenatal care, birth restrictions and protective measures.

\section{Psychological responses}

Participants reported a wide range of psychological responses to the pandemic: anxiety, grief, mourning, sadness, fear, irritation, nervousness, anger and feeling overwhelmed. Some also reported panic attacks and insomnia. The central subthemes in the psychological responses were anxiety and grief.

\section{Anxiety and grief}

Participants reported multiple worries, including anxiety about being infected with SARS-CoV-2; transmission of the virus to the fetus; and a lack of clear evidence about the effect of COVID19 on pregnancy and the developing fetus. Some worries were related to health services, such as the risks associated with a hospital birth and their impact on birth plans; the availability of health services if required; or the inability to take medication during pregnancy if they became ill. Others were related to the social effects of the pandemic, such as being isolated; the effect of social distancing and related measures on overall well-being during pregnancy and early motherhood; the long-term effects of COVID-19 on infants and children; the inability to develop social connections and support systems because of the pandemic; increased child care obligations; reduced instrumental support (e.g., help with child care, meal preparation, errands) because of social distancing and travel restrictions; and the inability to deal with stress and anxiety in conventional ways (e.g., exercise and visiting friends and family). Some reported worries related to job security, unemployment and the inability to accumulate required work hours to be qualified for maternity leave; financial strains; and general anxiety about the future and related uncertainties.
Table 1: Participant characteristics $(n=96)$

Characteristic Value*

\begin{tabular}{ll}
\hline Age, yr & $31.70 \pm 3.92$
\end{tabular}

Gestational age at recruitment, wk $22.73 \pm 8.93$

Gender†

\begin{tabular}{lc} 
Female & $89(92.7)$ \\
\hline Male & 0 \\
\hline Nonbinary & $1(1.0)$ \\
\hline Racial or ethnic background: white & $70(72.9)$ \\
\hline Education &
\end{tabular}

Education

High school diploma, certificate or incomplete university

University degree

$34(35.4)$

$62(64.6)$

Married, common-law or live-in partner

94 (97.9)

Born in Canada

$74(77.1)$

Geographic region§

Fraser Health

$32(33.3)$

Vancouver Coastal Health

$28(29.2)$

Interior Health

$21(21.9)$

Island Health

6 (6.3)

Northern Health

$1(1.0)$

Household income

Under $\$ 40000$

$6(6.2)$

$\$ 40000$ to $\$ 99999$

37 (38.6)

$\$ 100000$ and above

53 (55.2)

Paid work during current pregnancy

89 (92.7)

Primigravida

$33(34.4)$

Multigravida

$63(65.6)$

Did not receive prenatal care as early as desired

Problems or complications during current

pregnancy

GAD-7 score

$5.76 \pm 4.19$

Anxiety symptoms

\begin{tabular}{lc} 
None (GAD-7 score < 5) & $46(47.9)$ \\
\hline Mild (GAD-7 score 5-9) & $33(34.4)$ \\
\hline Moderate (GAD-7 score 10-14) & $13(13.5)$ \\
\hline Severe (GAD-7 score > 14) & $4(4.2)$ \\
\hline EPDS score & $7.31 \pm 3.97$ \\
Depressive symptoms & $62(64.6)$ \\
No (EPDS score < 10) & $34(35.4)$ \\
Yes (EPDS score $\geq 10)$ &
\end{tabular}

Note: EPDS = Edinburgh Postnatal Depression Scale, GAD-7 = 7-item

Generalized Anxiety Disorder scale, SD = standard deviation.

${ }^{*}$ Mean \pm SD or no. (\%).

†Missing data for 6 participants $(6.3 \%)$.

$\ddagger$ Values for other ethnic groups were very small and are not shown here for privacy reasons.

§Missing data for 8 participants $(8.3 \%)$. 
Table 2 (part 1 of 3): Summary of themes from qualitative data and representative quotes (Mar. 20 to May 31, 2020; British Columbia, Canada)

Theme/category

Representative quote

Responses to the COVID-19 pandemic

Psychological responses

Anxiety and grief

"It's had a major impact mentally and physically ... I had my anxiety in check, it was well managed. Now I feel anxious every day. I've felt panic. I'm teary and emotional."(Participant 28, Mar. 29)

"It is something I think about and get frustrated about ... why does it have to happen during my pregnancy?" (Participant 6, Apr. 1)

"I have been sad most days, as this was not how we planned for the pregnancy to go. After $5 \frac{1}{2}$ years of trying to get pregnant, we really wanted to celebrate this baby; this will now change our plans." (Participant 38, Apr. 6)

"It had a huge impact 2 months ago, when it began. Mid-March when everything was ramping up and the world was changing. That was also when we had our detailed ultrasound and the small pleural effusion was noted. I just completely ran out of any ability to cope. I think I had a panic attack. I couldn't stop worrying, crying. And of course then I worried about my mental state having an effect on the baby. I'm happy to say l've been doing a lot better since the end of March. It feels like we adapted to this new reality. We're healthy and safe. The pleural effusion is gone; baby is healthy and safe. I feel much more calm and steady now." (Participant 71, May 11)

"It has impacted my mental health. I'm a bit better this month, but I was really stressed out in March and parts of April while I was in the first trimester. I'm not coping with the uncertainty well, and I'm upset because these are my last few months of being in a childless couple and I'm not getting to savour our freedom as much as l'd like." (Participant 80, May 13)

"Definitely increased anxiety in a lot of areas I never would have had anxiety prepandemic (hospital setting, will my spouse be allowed in, what will my quality of care look like in hospital, will they be rushing me out before l'm ready) - all thoughts of unknown and worry around these factors increasing anxiety." (Participant 92, May 23)

"It [pandemic] has definitely impacted me and my experience with pregnancy. It has taken away many of the experiences I was looking forward to, removed the majority of human-human interaction from my life and placed a whole lot of uncertainty on a time already filled with so many physical and mental changes. Only time will tell if or how it impacts my baby. I can only hope this doesn't continue throughout baby's first year of life." (Participant 89, May 28)

Responses to pandemic-imposed prenatal care, birth restrictions and protective measures

Loss of support and connections

Loss of control and autonomy
"My partner is no longer allowed to attend our OB [obstetrician] appointments due to COVID, and his emotional support has been very important to me during those appointments, because I have a phobia of doctors and often forget what the doctor says, or I forget to ask the questions I need to ask. Not having him there to see the ultrasounds or hear the heartbeat interferes with his ability to attach, and our shared experience of attachment with our baby." (Participant 13, Mar. 27) "My boys were looking forward to the ultrasound, but I wasn't allowed any extra people due to precautions. I cried during the ultrasound." (Participant 53, Apr. 24)

"The uncertainty of restrictions at the hospital makes me feel a loss of control and has significantly impacted my anxiety (i.e., will the doula we paid for be allowed, how will we safely leave the hospital to minimize exposure, how clean will the staff and room be, will there be adequate staffing, will me and baby be in danger, will I have less choice or control due to these restrictions?)" (Participant 13, Mar. 27)

"Fear of the unknown [and] lack of control and freedom contribute to the return of moderate anxiety and depression, feelings of being mentally trapped. I feel [like] I'm grieving, losing some freedom." (Participant 28, Mar. 29)

"I feel slightly worried and a bit angry over choices that have been taken away from me, such as having a support person present during my prenatal and ultrasound appointments." (Participant 30, Apr. 9)

"I [am] also nervous about everyone being in masks and goggles while l'm delivering; not very personal." (Participant 35, Apr. 10)
Similarly, quantitative data showed prevalent anxiety and depressive symptoms among pregnant people. Of the participant sample, 54\% $(n=50)$ reported anxiety symptoms ranging from mild to severe as measured using GAD7. Depressive symptoms were reported by $35 \%$ of participants $(n=34)$.
Numerous participants identified a sense of loss and grief about losing a "normal" pregnancy experience and expressed irritation that a "normal" experience had been taken from them. Several participants who answered open-ended questions later in the study period described being very tense at the beginning of the pandemic in March, a state that had gradually eased by May. 
Table 2 (part 2 of 3): Summary of themes from qualitative data and representative quotes (Mar. 20 to May 31, 2020; British Columbia, Canada)

\begin{tabular}{|c|c|}
\hline Theme/category & Representative quote \\
\hline \multicolumn{2}{|c|}{ Experiences related to the pandemic } \\
\hline $\begin{array}{l}\text { Uncertainty about birth } \\
\text { plans and setting }\end{array}$ & $\begin{array}{l}\text { "I am scared how the virus will impact our plan for a hospital birth and what risks are going to be } \\
\text { associated with a hospital birth." (Participant } 7, \text { Mar. 29) } \\
\text { "I wanted to possibly have a home birth before this pandemic, but now I am fully planned on } \\
\text { delivering at home. I worry that I may have to go to hospital with baby even if I deliver at home ... } \\
\text { What if I tear, bleeding doesn't stop etc. I don't want to bring baby into a hospital." (Participant } 28, \\
\text { Mar. 29) }\end{array}$ \\
\hline $\begin{array}{l}\text { Added burden to existing } \\
\text { health and social disparities }\end{array}$ & $\begin{array}{l}\text { "We also don't have a vehicle and have been told to avoid public transit and car shares; so for my } \\
\text { biweekly OB appointments, I'm needing to walk an hour each way, which is exposing me to } \\
\text { others I pass on the sidewalk or bridge (which worries me), and it is also hard on my body at this } \\
\text { stage in [the] pregnancy." (Participant } 13 \text {, Mar. } 27 \text { ) }\end{array}$ \\
\hline $\begin{array}{l}\text { Perceived or projected lack } \\
\text { of support or limited support }\end{array}$ & $\begin{array}{l}\text { "The vision of introducing our baby to our closest friends and family in person will now be by } \\
\text { FaceTime, which is depressing. I worry how a lack of socialization will impact the baby over time." } \\
\text { (Participant 7, Mar. 29) } \\
\text { "Allow more than one person during my delivery. I need both my husband and my mom, and I'm } \\
\text { being forced to choose one. It's horrible to put people in these positions." (Participant } 58, \text { Apr. 24) } \\
\text { "We can't even plan how we are going to manage maternity or paternity leave, because my } \\
\text { husband works out of province and flies, so he is in a higher risk category according to my doctor } \\
\text { and we don't know if he can keep working or will need to take time off so he can bond with baby. If } \\
\text { his camp has an outbreak near my due date, he could be put on another } 14 \text {-day self-isolation and } \\
\text { miss the birth. He will be taking over a month of holidays, but if baby comes early it could be in a } \\
\text { possible isolation window and l'll have to do it without him ... I am alone } 50 \% \text { of every month while } \\
\text { husband works. We are in separate beds and bathrooms } 25 \% \text { of every month for } 1 \text { of his } 2 \text { weeks } \\
\text { at home as a precaution due to his camp work and flights, and try to get back to normal the last } \\
25 \% \text { of each month ... Especially my in-laws work in health care or have been continuing to meet } \\
\text { with other for 'distancing walks' or 'distant visits,' which I don't think entirely follow the safety } \\
\text { recommendations, and l'm worried they won't take the risk seriously enough and I will become a } \\
\text { bad guy for protecting my newborn by keeping the baby away from those who I don't think are } \\
\text { keeping safe enough. I am already judged for being paranoid or taking it too seriously, but many } \\
\text { pregnant friends or friends with little ones feel the same. I plan to put my little one's safety first if } \\
\text { needed, even at the expense of those other relationships, which sucks." (Participant } 89 \text {, May 28) }\end{array}$ \\
\hline $\begin{array}{l}\text { Concerns about early } \\
\text { development }\end{array}$ & $\begin{array}{l}\text { "I feel worried about how the stress is impacting my developing baby. If we were not in this } \\
\text { current situation, my stress and anxiety levels would be much lower. I could manage my stress in } \\
\text { normal ways like meeting up with friends or taking my children to go do something fun. Instead, it } \\
\text { feels like constant stress with no break or end in sight." (Participant 32, Apr. 11) }\end{array}$ \\
\hline $\begin{array}{l}\text { Struggles over managing } \\
\text { multiple demands }\end{array}$ & $\begin{array}{l}\text { "... has increased my anxiety. Concerned about working at my job ... Concerned that I will } \\
\text { contract the virus and there have been few studies identifying whether it is passed through } \\
\text { vertical transmission, harm to fetus." (Participant 15, Mar. 25) } \\
\text { "It (the pandemic) has certainly caused stressed stemming from work-life balance. My workload } \\
\text { is mounting as I have fewer hours in the day to work, trying to care for my child at home now. } \\
\text { When I am working, I feel guilty l'm not focused on my child and when I'm not working, I feel } \\
\text { stressed about falling more behind." (Participant 18, Apr. 2) } \\
\text { "Work is more challenging. PPE [personal protective equipment] makes nausea challenging ... } \\
\text { It's [also] hard sitting with [the] pregnant people in my care through their anxieties." (Participant } \\
43, \text { Apr. 17) }\end{array}$ \\
\hline
\end{tabular}

Responses to pandemic-imposed prenatal care, birth restrictions and protective measures

Participants reported the implementation of restrictions related to their pregnancy care, including not being allowed to have a support person during prenatal care or ultrasound visits, or being allowed only a limited number of support people during labour.

\section{Loss of support and connections}

Participants reported a loss of support during pregnancy or a projected loss of support during labour because of pandemicrelated restrictions. They expressed concerns that these restrictions had jeopardized or would jeopardize the quality of their pregnancy and birth experience. Participants often noted that the new restrictions negatively affected the support and connections they had hoped to receive and develop.

\section{Loss of control and autonomy}

Having limited choices as a result of the restrictions and not knowing what to expect during the birthing process contributed to a sense of loss of control. Some participants commented that uncertainty about the birthing process compromised their sense of control and provoked anxiety. Irritation, 
Table 2 (part 3 of 3): Summary of themes from qualitative data and representative quotes (Mar. 20 to May 31, 2020; British Columbia, Canada)

Theme/category

Representative quote

Perceived maternity care needs

Positive experiences:

a supportive system

"My health care provider has answered my questions about the hospital well and has found adaptive and creative ways to ensure I had the information I needed. At the midwifery-family doctor maternity care clinic I attend, they offer group classes for the end of the second trimester and beginning of the third trimester. When those were cancelled due to COVID-19, they created a condensed Zoom version that was really helpful. They also provided the typical hospital tour (which was now not allowed) by YouTube video. Both of these efforts helped to reduce uncertainty and provide knowledge and familiarity with the experience to come. It helped reduce some of my nervousness about labour." (Participant 90, May 26)

"My doctor has been amazing. She is new to me (since week 17) and has called me every few weeks to chat, even before the pandemic broke out in Canada. She has provided me with protocols and procedures so that I feel prepared should I contract COVID. She has provided me with update research of the realities of the impact that COVID-19 has on pregnant mothers and babies, and also given me various reliable social media pages to be following." (Participant 46, Apr. 22)

Maintaining prenatal care "I understand why in-person appointments aren't possible now, but it is really hard to feel visits connected to health care providers over the phone. I think video calls work better." (Participant 73 , May 13)

"My midwife appointments have been monthly, alternating between online and in person. I haven't felt the need for additional check-ins; however, I'm sure some people would." (Participant 86, May 19)

"Keeping visits as normal as possible. More in person and less telehealth would be nice, but I understand it's not reasonable at this point." (Participant 94, May 25)

Offering the option of

in-person visits

"The prenatal appointment schedule includes a lot less frequent visits, which worries me that things could get missed." (Participant 20, Apr. 1)

"My maternity doctor still sees me in person, which I really appreciate, and it's been a big support for me." (Participant 76, May 20)

Frequent and proactive check-ins to build rapport

"I think I am feeling a lack of connection to them. I had hoped to be building stronger relationships with them so I feel more comfortable with them during my delivery." (Participant 78, May 12) "More check-ins would be nice. We have no appointments until 20 weeks after initial intake." (Participant 74, May 13)

"More regular check-ins via email or phone call. Providing available resources for those who aren't aware of them." (Participant 88, May 20)

"More regular check-ins. I still have appointments with the midwife but no family doctor or any other health care provider to check in during those 1 to 1.5 months with no appointment. The health coverage I have for counselling is not great, so I have not been talking with a professional." (Participant 82, May 26)

Mental health support $\quad$ "They can emotionally support their patients." (Participant 27, Apr. 2)

"Setting up a counselling or psychology appointment." (Participant 63, May 9)

Partner engagement

"They could let me take a video of my ultrasound so I can actually share it with my husband since he's not allowed to join me at my ultrasound appointments and missed the last one. Hospital policy wouldn't even let me FaceTime him live for a moment to show him the movement and heartbeat at 20 weeks. It really sucked not being able to share that with anyone, even though the tech was lovely and got some great pics." (Participant 89, May 28)

anxiety and worry related to the restrictions were a recurring theme. It was evident that the pandemic-imposed maternity care constraints had created additional anxiety for participants, who were already anxious. Some described the effect of these restrictions as "losing some freedom." Concerns also emerged about an unfriendly and impersonal hospital environment because of personal protective measures.

\section{Experiences related to the pandemic}

The theme of people's experiences related to the pandemic included uncertainty about birth plans and birth settings; added burden to the existing health and social disparities; perceived or projected lack of support or limited support; concerns about early development; and struggles over managing multiple demands.

\section{Uncertainty about birth plans and birth settings}

Several participants reported feeling uncertain about the safety of hospitals and expressed fear of giving birth in a hospital because of the possibility of contracting COVID-19. A few participants commented that they were considering home births because of these concerns. Two participants reported that although they had confirmed plans for a home birth, they were still fearful that at some point during labour they might have to be transferred to a hospital. 
Added burden to existing health and social disparities

Some participants reported anxiety about being infected because of frequent pharmacy or hospital visits related to pregnancy and its complications. Participants with limited resources and new immigrants also reported additional stress and burden. For instance, one participant reported that she did not own a vehicle and had been advised not to use public transit; as a result, she had to walk long distances to attend her prenatal appointments.

Perceived or projected lack of support or limited support Some participants described the expectation of limited support around labour as daunting. They expressed concern about uncertainties related to child care when they went into labour, having a complicated or cesarean delivery and recovery, and whether close family members would be available to support them, considering the restrictions on travel and social distancing. Mothers expecting a second or third child also expressed worries about being overwhelmed while self-isolating and not having support from partners, who may or may not have been able to take time off work for financial reasons.

\section{Concerns about early development}

Participants expressed concerns about how isolation and social distancing would affect their child. They also reported concerns about their own elevated anxiety during pregnancy and its potential consequences for the developing fetus.

\section{Struggles over managing multiple demands}

Most participants (over 90\%) had a paid job during pregnancy, and some reported stress about multitasking and worklife balance. Several participants were front-line workers or partners of front-line workers; an additional layer of anxiety was evident among these participants.

\section{Perceived maternity care needs}

We asked participants how their health care providers could support them better during the pandemic. Several participants reported that they had found their health care providers to be supportive and understanding. Participants also identified several health care needs, outlined below.

\section{Maintaining prenatal care visits}

Some participants reported interruptions in their maternity care services, such as cancelled appointments or a reduced number of visits early in the pandemic. They identified a need to maintain their prenatal care visits and for their care providers to provide the latest information about the specific effects of COVID-19 on pregnancy. Several participants also expressed worries about the potential implications of the next waves of the pandemic for their health care.

\section{Offering the option of in-person visits}

Many participants reported receiving virtual care. Because of less frequent or virtual visits, some participants reported concerns that some health issues might not be identified.

\section{Frequent and proactive check-ins to build rapport}

Because of cancelled, less frequent or short virtual appointments, some participants reported feeling a lack of connection with their maternity care providers. They also noted that they experienced difficulty in building rapport with their providers through virtual care. They stated that more frequent contact would help them develop relationships with their health care providers, so that they would feel more comfortable during labour. They also noted that the increased availability of their providers for more phone check-ins, answering questions and some proactive check-ins would be helpful.

\section{Mental health support}

Most participants reported a substantial need for mental health support in the form of "check-ins," coping advice for pandemic-related anxieties and constraints, and setting up counselling and psychology appointments.

\section{Partner engagement}

Some participants suggested engaging partners and educating them on how to support pregnant people, particularly because partners were not often allowed during prenatal care or ultrasound visits.

\section{Interpretation}

This study provides insights into the experiences of pregnant people, their responses to the COVID-19 pandemic and their perceived maternity care needs between Mar. 20 and May 31, 2020, in BC. The launch of data collection for the qualitative component just after the World Health Organization had declared COVID-19 to be a pandemic ${ }^{26}$ enabled us to explore and understand the experiences and responses of pregnant people during the first pandemic wave. Qualitative quotations and quantitative measures of anxiety symptoms from our sample indicated highly elevated anxiety symptoms at the onset of the pandemic. Several participants reported that these anxieties gradually eased as the number of new cases in the community decreased.

In the present study, pregnant people reported multiple stressors related to the pandemic, and 44\% reported anxiety symptoms. Previous Canadian community-based studies with similar inclusion criteria reported anxiety symptoms at a rate of $15 \%$ to $19 \% .{ }^{27}$ Similar findings of poor maternal mental health during the pandemic have been reported globally. ${ }^{7,28-30}$ The most dominant psychological responses in our study were anxiety and grief, indicating that mental health support, consultations and interventions should be focused on these issues.

Because the pandemic caused major shifts in hospital routines, some participants reported that uncertainty about birth plans and not knowing what to expect during a hospital visit provoked or intensified their anxiety. Providing more tangible information about new hospital birthing processes (e.g., offering a virtual tour of the labour and delivery unit to demonstrate pandemic-related modifications and details on 
personal protection measures and safety) could help pregnant people become familiar with the new environment, prepare mentally and gain confidence about the safety of a hospital birth. This information could serve to reduce anxiety about the unknown components of the birthing process during the pandemic.

Although restrictions and protective measures have been implemented to protect patients, study participants noted that these measures also imposed additional burdens by limiting access to support and diminishing their sense of control and autonomy during pregnancy and the birthing process. Data from previous pandemics have shown that outbreaks can jeopardize the capacity of health care services in general. ${ }^{15}$ There is some emerging evidence suggesting a potential increase in labour interventions among people with COVID-19., ${ }^{43}$ In a recent systematic review of 6 studies of 51 pregnant people diagnosed with COVID-19, almost all of them had a cesarean delivery, frequently before term, and without any clear indication for surgery. Although COVID19 may be associated with spontaneous preterm birth, it has been suggested that the increased risk of preterm birth is a consequence of elective interventions carried out as a precautionary measure. ${ }^{13}$ Early in the pandemic, some hospitals adopted policies that could have increased labour interventions. ${ }^{31,32}$ However, a 2021 systematic review did not find an overall change in labour induction and cesarean delivery rates during the pandemic among noninfected pregnant people. ${ }^{7}$

Many participants expressed concerns about their babies and whether social isolation would affect early development. Although evidence about the effect of pandemics on child development is scarce, disaster studies show that adverse changes in maternal mental health after a disaster strongly influence child development ${ }^{33}$ and affect the early development of children to a greater extent than the disaster itself. ${ }^{34}$ Participants also reported concerns about how their own mental health challenges and stresses related to the pandemic would influence the growth and development of their fetus. There is evidence showing that poor perinatal mental health is linked to higher rates of low birth weight and preterm birth, ${ }^{35-37}$ as well as poor cognitive, behavioural and psychomotor development and mental health problems in children. ${ }^{35,38-42}$ Further research is needed to elucidate the impact of this pandemic and associated social distancing on short-term and long-term developmental trajectories.

\section{Limitations}

This study had several limitations: it was a small study conducted in a single geographical area, and the findings might be applicable only to settings with a similar outbreak intensity. Findings were based on a convenience sample, so there is a possibility of selection bias. We did not collect the COVID-19 status of participants. We measured anxiety and depressive symptoms using self-reported screening tools; findings do not indicate a diagnosis of these mental health conditions. The open-ended questions were developed by the research team; no pilot study using these questions was conducted. The survey was not anonymous. Most of the participants were well educated, white and partnered; these characteristics could have limited the transferability of the findings. We have provided details of the participants' characteristics to enable readers to determine the applicability of our study findings to their populations.

\section{Conclusion}

In the present study, we found that the impact of the COVID-19 pandemic on the perinatal population has been substantial. The findings of this mixed-methods descriptive study provide some insights into the effect of the pandemic on this population and can help in planning informed and evidence-based health care interventions to mitigate adverse effects and support mothers and families. Further research is required to understand the short- and long-term physical, psychological and developmental effects of the COVID-19 pandemic on the perinatal population.

\section{References}

1. Tucci V, Moukaddam N, Meadows J, et al. The forgotten plague: psychiatric manifestations of Ebola, Zika, and emerging infectious diseases. 7 Glob Infect Dis 2017;9:151-6.

2. Madhav N, Oppenheim B, Gallivan M. Pandemics: risks, impacts, and mitigation. In: Jamison D, Gelband H, Horton S, editors. Disease control priorities: improving health and reducing poverty. 3rd ed. Washington (DC): International Bank for Reconstruction and Development / World Bank; 2017: chapter 17.

3. Zhao X, Jiang Y, Zhao Y, et al. Analysis of the susceptibility to COVID-19 in pregnancy and recommendations on potential drug screening. Eur 7 Clin Microbiol Infect Dis 2020;39:1209-20.

4. Di Mascio D, Khalil A, Saccone G, et al. Outcome of coronavirus spectrum infections (SARS, MERS, COVID-19) during pregnancy: a systematic review and meta-analysis. Am 7 Obstet Gynecol MFM 2020;2:100107.

5. Knight M, Bunch K, Vousden N, et al. Characteristics and outcomes of pregnant women admitted to hospital with confirmed SARS-CoV-2 infection in UK: national population-based cohort study. BMF 2020;369:m2107.

6. Sentilhes L, De Marcillac F, Jouffrieau C, et al. Coronavirus disease 2019 in pregnancy was associated with maternal morbidity and preterm birth. Am $\mathcal{f}$ Obstet Gynecol 2020; 223:914.e1-914.e15.

7. Chmielewska B, Barratt I, Townsend R, et al. Effects of the COVID-19 pandemic on maternal and perinatal outcomes: a systematic review and metaanalysis. Lancet Glob Health 2021;9:e759-e772.

8. Lee DT, Sahota D, Leung TN, et al. Psychological responses of pregnant women to an infectious outbreak: a case-control study of the 2003 SARS outbreak in Hong Kong. 7 Psychosom Res 2006;61:707-13.

9. Zorrilla C, Rivera-Vinas J, Garcia-Coll C, et al. Stress, depression and anxiety in pregnancy during the Zika epidemic in Puerto Rico: the case for group prenatal care. In: Proceedings of the first international conference on Zika virus; 2017 Feb. 22-25; Washington (DC). Cranbury (NJ): MJH Life Sciences; 2017.

10. Beigi RH. Pandemic influenza and pregnancy: a call for preparedness planning. Obstet Gynecol 2007;109:1193-6.

11. Villar J, Ariff S, Gunier RB, et al. Maternal and neonatal morbidity and mortality among pregnant women with and without COVID-19 infection: the INTERCOVID multinational cohort study. FAMA Pediatr 2021;175:817-26.

12. Harris J. Influenza occurring in pregnant women. $7 A M A$ 1919;72:3.

13. Della Gatta AN, Rizzo R, Pilu G, et al. Coronavirus disease 2019 during pregnancy: a systematic review of reported cases. Am 7 Obstet Gynecol 2020;223:36-41.

14. Giving birth in Canada: the costs. Ottawa: Canadian Institute for Health Information; 2006. Available: https://secure.cihi.ca/free_products/Costs_ Report_06_Eng.pdf (accessed 2021 Apr. 6).

15. Rasmussen SA, Jamieson DJ, Macfarlane K, et al. Pandemic influenza and pregnant women: summary of a meeting of experts. Am 7 Public Health 2009;99(Suppl 2):S248-54.

16. Morgan DL. Paradigms lost and pragmatism regained: methodological implications of combining qualitative and quantitative methods. 7 Mixed Methods Res 2007;1:48-76.

17. Tong A, Sainsbury P, Craig J. Consolidated criteria for reporting qualitative research (COREQ): a 32-item checklist for interviews and focus groups. Int 7 Qual Health Care 2007;19:349-57.

18. Cox JL, Holden JM, Sagovsky R. Detection of postnatal depression. Development of the 10-item Edinburgh Postnatal Depression Scale. Br 7 Psychiatry 1987;150:782-6. 
19. Eberhard-Gran M, Eskild A, Tambs K, et al. The Edinburgh Postnatal Depression Scale: validation in a Norwegian community sample. Nord $\mathcal{F}$ Psychiatry 2001;55:113-7.

20. Spitzer RL, Kroenke K, Williams JB, et al. A brief measure for assessing generalized anxiety disorder: the GAD-7. Arch Intern Med 2006;166:1092-7.

21. IBM SPSS Statistics for Windows. Version 19.0. Armonk (NY): IBM Corp; 2010.

22. Sandelowski M. Whatever happened to qualitative description? Res Nurs Health 2000;23:334-40.

23. Boyatzis RE. Transforming qualitative information: thematic analysis and code development. Thousand Oaks (CA): Sage; 1998.

24. Lincoln YS, Guba EG. Naturalistic inquiry. Beverly Hills (CA): Sage; 1985.

25. Sandelowski M. Rigor or rigor mortis: the problem of rigor in qualitative research revisited. ANS Adv Nurs Sci 1993;16:1-8.

26. WHO Director-General's opening remarks at the media briefing on COVID19, 11 March 2020. Geneva: The World Health Organization; 2020. Available: www.who.int/director-general/speeches/detail/who-director-general-s-opening -remarks-at-the-media-briefing-on-covid-19---11-march-2020 (accessed 2021 Apr. 6).

27. Bayrampour H, Salmon C, Vinturache A, et al. Effect of depressive and anxiety symptoms during pregnancy on risk of obstetric interventions. 7 Obstet Gynaecol Res 2015:41:1040-8.

28. Mappa I, Distefano FA, Rizzo G. Effects of coronavirus 19 pandemic on maternal anxiety during pregnancy: a prospectic observational study. 7 Perinat Med 2020;48:545-50.

29. Taubman-Ben-Ari O, Chasson M, Abu Sharkia S, et al. Distress and anxiety associated with COVID-19 among Jewish and Arab pregnant women in Israel. 7 Reprod Infant Psychol 2020;38:340-8.

30. Durankus F, Aksu E. Effects of the COVID-19 pandemic on anxiety and depressive symptoms in pregnant women: a preliminary study. 7 Matern Fetal Neonatal Med 2020 May 18 [Epub ahead of print]. doi: 10.1080/14767058.2020.1763946.

31. Drandic D, van Leeuwen F. "But a small price to pay" - degradation of rights in childbirth during COVID-19. Oxford (UK): Oxford Human Rights Hub, Faculty of Law, University of Oxford; 2020.

32. Laucius J. Almonte General Hospital requests all women in labour have an epidural to curb spread of COVID-19. Ottawa Citizen 2020 Apr. 10.

33. Harville E, Xiong X, Buekens P. Disasters and perinatal health: a systematic review. Obstet Gynecol Surv 2010;65:713-28.

34. Tees MT, Harville EW, Xiong X, et al. Hurricane Katrina-related maternal stress, maternal mental health, and early infant temperament. Matern Child Health 7 2010;14:511-8.

35. Field T. Prenatal anxiety effects: a review. Infant Behav Dev 2017;49:120-8.

36. Kramer MS, Lydon J, Seguin L, et al. Stress pathways to spontaneous preterm birth: the role of stressors, psychological distress, and stress hormones. Am 7 Epidemiol 2009;169:1319-26.
37. Liou SR, Wang P, Cheng CY. Effects of prenatal maternal mental distress on birth outcomes. Women Birth 2016;29:376-80.

38. Davis EP, Sandman CA. Prenatal psychobiological predictors of anxiety risk in preadolescent children. Psychoneuroendocrinology 2012;37:1224-33.

39. Loomans EM, van der Stelt O, van Eijsden M, et al. High levels of antenatal maternal anxiety are associated with altered cognitive control in five-year-old children. Dev Psychobiol 2012;54:441-50.

40. Buss C, Davis EP, Hobel CJ, et al. Maternal pregnancy-specific anxiety is associated with child executive function at 6-9 years age. Stress 2011;14:665-76.

41. Blair MM, Glynn LM, Sandman CA, et al. Prenatal maternal anxiety and early childhood temperament. Stress 2011;14:644-51.

42. O'Donnell KJ, Glover V, Barker ED, et al. The persisting effect of maternal mood in pregnancy on childhood psychopathology. Dev Psychopathol 2014;26:393-403

Affiliations: Department of Family Practice (Bayrampour, Tamana) and of Obstetrics and Gynecology (Boutin), Faculty of Medicine, University of British Columbia, Vancouver, BC

Contributors: Hamideh Bayrampour conceptualized the study, acquired, analyzed and interpreted the data and drafted the manuscript. Sukhpreet Tamana acquired, analyzed and interpreted the data and revised the manuscript for important intellectual content. Amelie Boutin contributed to the interpretation of data and revised the manuscript for important intellectual content. All authors approved the final version of the manuscript for publication and agree to be responsible for its content.

Funding: The original Pregnancy Specific Anxiety Scale study was funded by the Canadian Institutes for Health Research. There was no funding source for this specific substudy.

Content licence: This is an Open Access article distributed in accordance with the terms of the Creative Commons Attribution (CC BY-NC-ND 4.0) licence, which permits use, distribution and reproduction in any medium, provided that the original publication is properly cited, the use is noncommercial (i.e., research or educational use), and no modifications or adaptations are made. See: https://creativecommons.org/licenses/ by-nc-nd/4.0/

Data sharing: Data are not publicly available.

Supplemental information: For reviewer comments and the original submission of this manuscript, please see www.cmajopen.ca/content /10/1/E146/suppl/DC1. 